\title{
ON SOME PROPERTIES OF $\oplus$-SUPPLEMENTED MODULES
}

\author{
A. IDELHADJ and R. TRIBAK
}

Received 15 March 2002

\begin{abstract}
A module $M$ is $\oplus$-supplemented if every submodule of $M$ has a supplement which is a direct summand of $M$. In this paper, we show that a quotient of a $\oplus$-supplemented module is not in general $\oplus$-supplemented. We prove that over a commutative ring $R$, every finitely generated $\oplus$-supplemented $R$-module $M$ having dual Goldie dimension less than or equal to three is a direct sum of local modules. It is also shown that a ring $R$ is semisimple if and only if the class of $\oplus$-supplemented $R$-modules coincides with the class of injective $R$-modules. The structure of $\oplus$-supplemented modules over a commutative principal ideal ring is completely determined.
\end{abstract}

2000 Mathematics Subject Classification: 16D50, 16D60, 13E05, 13E15, 16L60, 16P20, 16D80.

1. Introduction. All rings considered in this paper will be associative with an identity element. Unless otherwise mentioned, all modules will be left unitary modules. Let $R$ be a ring and $M$ an $R$-module. Let $A$ and $P$ be submodules of $M$. The submodule $P$ is called a supplement of $A$ if it is minimal with respect to the property $A+P=M$. Any $L \leq M$ which is the supplement of an $N \leq M$ will be called a supplement submodule of $M$. If every submodule $U$ of $M$ has a supplement in $M$, we call $M$ complemented. In [25, page 331], Zöschinger shows that over a discrete valuation ring $R$, every complemented $R$-module satisfies the following property $(P)$ : every submodule has a supplement which is a direct summand. He also remarked in [25, page 333] that every module of the form $M \cong\left(R / a_{1}\right) \times \cdots \times\left(R / a_{n}\right)$, where $R$ is a commutative local ring and $a_{i}(1 \leq i \leq n)$ are ideals of $R$, satisfies $(P)$. In [12, page 95], Mohamed and Müller called a module $\oplus$-supplemented if it satisfies property $(P)$.

On the other hand, let $U$ and $V$ be submodules of a module $M$. The submodule $V$ is called a complement of $U$ in $M$ if $V$ is maximal with respect to the property $V \cap U=0$. In [17] Smith and Tercan investigate the following property which they called $\left(C_{11}\right)$ : every submodule of $M$ has a complement which is a direct summand of $M$. So, it was natural to introduce a dual notion of $\left(C_{11}\right)$ which we called $\left(D_{11}\right)$ (see $\left.[6,7]\right)$. It turns out that modules satisfying $\left(D_{11}\right)$ are exactly the $\oplus$-supplemented modules. A module $M$ is called a completely $\oplus$-supplemented (see [5]) (or satisfies $\left(D_{11}^{+}\right)$in our terminology, see [6, 7]) if every direct summand of $M$ is $\oplus$-supplemented. 
Our paper is divided into four sections. The purpose of Section 2 is to answer the following natural question: is any factor module of a $\oplus$-supplemented module $\oplus$-supplemented? Some relevant counterexamples are given.

In Section 3 we prove that, over a commutative ring, every finitely generated $\oplus$-supplemented module having dual Goldie dimension less than or equal to three is a direct sum of local modules.

Section 4 describes the structure of $\oplus$-supplemented modules over commutative principal ideal rings.

In the last section we determine the class of rings $R$ with the property that every $\oplus$-supplemented $R$-module is injective. These turn out to be the class of all left Noetherian $V$-rings (Proposition 5.3). It is also shown that a ring $R$ is semisimple if and only if the class of $\oplus$-supplemented $R$-modules coincides with the class of injective $R$-modules (Proposition 5.5).

For an arbitrary module $M$, we will denote by $\operatorname{Rad}(M)$ the Jacobson radical of $M$. The injective hull of $M$ will be denoted by $E(M)$. The annihilator of $M$ will be denoted by $\operatorname{Ann}_{R}(M)$. A submodule $A$ of $M$ is called small in $M(A \ll M)$ if $A+B \neq M$ for any proper submodule $B$ of $M$. A nonzero module $H$ is called hollow if every proper submodule is small in $H$ and is called local if the sum of all its proper submodules is also a proper submodule. We notice that a local module is just a cyclic hollow module.

2. Quotients of $\oplus$-supplemented modules. By [23, corollary on page 45], every factor module of a complemented module is complemented. Now, let $M$ be a $\oplus$-supplemented module. In this section we will answer the following natural question: is any factor module of $M \oplus$-supplemented?

First, we mention the following result, which we will use frequently in the sequel.

Proposition 2.1 [6, Proposition 1]. The following are equivalent for a module $M$ :

(i) $M$ is $\oplus$-supplemented;

(ii) for any submodule $N$ of $M$, there exists a direct summand $K$ of $M$ such that $M=N+K$ and $N \cap K$ is small in $K$.

A commutative ring $R$ is a valuation ring if it satisfies one of the following three equivalent conditions:

(i) for any two elements $a$ and $b$, either $a$ divides $b$ or $b$ divides $a$;

(ii) the ideals of $R$ are linearly ordered by inclusion;

(iii) $R$ is a local ring and every finitely generated ideal is principal. A module $M$ is called finitely presented if $M \cong F / K$ for some finitely generated free module $F$ and finitely generated submodule $K$ of $F$. An important result about these modules is that if $M$ is finitely presented and $M \cong F / G$, where $F$ is a finitely generated free module, then $G$ is also finitely generated (see [2]). 
EXAMPLE 2.2. Let $R$ be a commutative local ring which is not a valuation ring and let $n \geq 2$. By [21, Theorem 2], there exists a finitely presented indecomposable module $M=R^{(n)} / K$ which cannot be generated by fewer than $n$ elements. By [6, Corollary 1], $R^{(n)}$ is $\oplus$-supplemented. However $M$ is not $\oplus$-supplemented [6, Proposition 2].

The dual Goldie dimension of an $R$-module, denoted by corank $\left({ }_{R} M\right)$, was introduced by Varadarajan in [19]. If $M=0$, the corank of $M$ is defined as 0 . Let $M \neq 0$ and $k$ an integer greater than or equal to one. If there is an epimorphism $f: M \rightarrow \prod_{i=1}^{k} N_{i}$, where each $N_{i} \neq 0$, we say that the $\operatorname{corank}\left({ }_{R} M\right) \geq k$. If $\operatorname{corank}\left({ }_{R} M\right) \geq k$ and $\operatorname{corank}\left({ }_{R} M\right) \nsucceq k+1$, then we define $\operatorname{corank}\left({ }_{R} M\right)=k$. If the corank $\left({ }_{R} M\right) \geq k$ for every $k \geq 1$, we say that the $\operatorname{corank}\left({ }_{R} M\right)=\infty$. It was shown in $[14,19]$ that the $\operatorname{corank}\left({ }_{R} M\right)<\infty$ if and only if there is an epimorphism $f: M \rightarrow \prod_{i=1}^{k} H_{i}$, where $H_{i}$ is hollow and $\operatorname{ker}(f)$ is small in $M$.

As in [20], a module $M$ has the exchange property if for any module $G$, where

$$
G=M^{\prime} \oplus C=\oplus_{i \in I} D_{i}
$$

with $M^{\prime} \cong M$, there are submodules $D_{i}^{\prime} \leq D_{i}$ such that $G=M^{\prime} \oplus\left(\oplus_{i \in I} D_{i}^{\prime}\right)$.

Before proceeding any further, we consider another example (note that the module considered is decomposable).

EXAMPLE 2.3. Let $R$ be a commutative local ring which is not a valuation ring. Let $a$ and $b$ be elements of $R$, neither of them divides the other. By taking a suitable quotient ring, we may assume $(a) \cap(b)=0$ and $a m=b m=0$, where $m$ is the maximal ideal of $R$. Let $F$ be a free module with generators $x_{1}, x_{2}$, and $x_{3}$. Let $K$ be the submodule generated by $a x_{1}-b x_{2}$ and let $M=F / K$. Thus,

$$
M=\frac{R x_{1} \oplus R x_{2} \oplus R x_{3}}{R\left(a x_{1}-b x_{2}\right)}=\left(R \overline{x_{1}}+R \overline{x_{2}}\right) \oplus R \overline{x_{3}} .
$$

Suppose that $M$ is $\oplus$-supplemented. There exist submodules $H$ and $N$ of $M$ such that $M=H \oplus N, R \overline{x_{1}}+N=M$, and $R \overline{x_{1}} \cap N$ is small in $N$ (Proposition 2.1). By the proof of [21, Theorem 2], $R \overline{x_{1}}+R \overline{x_{2}}$ is an indecomposable module which cannot be generated by fewer than 2 elements. Thus $\operatorname{corank}\left(R \overline{x_{1}}+R \overline{x_{2}}\right)=2$ by [14, Proposition 1.7]. Hence $\operatorname{corank}(M)=3$. Since $H \cong M / N$ and $M / N \cong$ $R \overline{x_{1}} /\left(N \cap R \overline{x_{1}}\right)$, we get that $H$ is a local direct summand of $M$ and hence $\operatorname{corank}(N)=2$ (see [14, Corollary 1.9]). Since $R$ is a commutative local ring, $\operatorname{End}_{R}\left(R \overline{x_{3}}\right)$ is a local ring by [4, Theorem 4.1]. Since $R \overline{x_{3}}$ has the exchange property [20, Proposition 1], there are submodules $H^{\prime} \leq H$ and $N^{\prime} \leq N$ such that $M=R \overline{x_{3}} \oplus H^{\prime} \oplus N^{\prime}$. Therefore $R \overline{x_{1}}+R \overline{x_{2}} \cong H^{\prime} \oplus N^{\prime}$. Thus $H^{\prime} \oplus N^{\prime}$ is indecomposable. Hence $N^{\prime}=0$ or $H^{\prime}=0$. But $\operatorname{corank}(M)=3$ and $\operatorname{corank}(N)=2$, so $M=R \overline{x_{3}} \oplus N$ and $N \cong R \overline{x_{1}}+R \overline{x_{2}}$ is indecomposable. Since $\overline{x_{1}}, \overline{x_{2}} \in M$, there are $\alpha, \beta \in R$ and $\overline{y_{1}}, \overline{y_{2}} \in N$ such that $\overline{x_{1}}=\alpha \overline{x_{3}}+\overline{y_{1}}$ and $\overline{x_{2}}=\beta \overline{x_{3}}+\overline{y_{2}}$. Hence $\overline{x_{1}}-\alpha \overline{x_{3}} \in N$ and $\overline{x_{2}}-\beta \overline{x_{3}} \in N$. But $M=R \overline{x_{3}} \oplus\left[R\left(\overline{x_{1}}-\alpha \overline{x_{3}}\right)+R\left(\overline{x_{2}}-\beta \overline{x_{3}}\right)\right]$. Then $N=R\left(\overline{x_{1}}-\alpha \overline{x_{3}}\right)+R\left(\overline{x_{2}}-\beta \overline{x_{3}}\right)$. Now, $M=R \overline{x_{1}}+N$ and $\overline{x_{3}} \in M$, so 
there exists $\alpha^{\prime} \in R$ such that $\overline{x_{3}}-\alpha^{\prime} \overline{x_{1}} \in N$. Note that $\alpha^{\prime} \overline{x_{1}}-\alpha^{\prime} \alpha \overline{x_{3}} \in N$ and $\left(1-\alpha^{\prime} \alpha\right) \overline{x_{3}} \in N \cap R \overline{x_{3}}$. Thus $\left(1-\alpha^{\prime} \alpha\right) \overline{x_{3}}=0$, that is, $\left(1-\alpha^{\prime} \alpha\right) x_{3} \in$ $R\left(a x_{1}-b x_{2}\right)$. Hence $1-\alpha^{\prime} \alpha=0$. So $\alpha$ is invertible and $\alpha^{-1}=\alpha^{\prime}$. Note that

$$
a\left(\overline{x_{1}}-\alpha \overline{x_{3}}\right)-b\left(\overline{x_{2}}-\beta \overline{x_{3}}\right)=(b \beta-a \alpha) \overline{x_{3}} .
$$

Thus $a\left(\overline{x_{1}}-\alpha \overline{x_{3}}\right)-b\left(\overline{x_{2}}-\beta \overline{x_{3}}\right) \neq 0$. Otherwise, $(b \beta-a \alpha) x_{3} \in R\left(a x_{1}-b x_{2}\right)$, which gives $b \beta=a \alpha$ and then $a=b \beta \alpha^{\prime}$, which is a contradiction. Since ( $b \beta-$ $a \alpha) \overline{x_{3}} \in N \cap R \overline{x_{3}}$, then $N \cap R \overline{x_{3}} \neq 0$, which is a contradiction. It follows that $M$ is not $\oplus$-supplemented. But $R x_{1} \oplus R x_{2} \oplus R x_{3}$ is completely $\oplus$-supplemented [6, Corollary 2].

These examples show that a factor module of a $\oplus$-supplemented module is not in general $\oplus$-supplemented.

Proposition 2.5 deals with a special case of factor modules of $\oplus$-supplemented modules. First we prove the following lemma.

LEMMA 2.4. Let $M$ be a nonzero module and let $U$ be a submodule of $M$ such that $f(U) \leq U$ for each $f \in \operatorname{End}_{R}(M)$. If $M=M_{1} \oplus M_{2}$, then $U=U \cap M_{1} \oplus U \cap M_{2}$.

Proof. Let $\pi_{i}: M \rightarrow M_{i}(i=1,2)$ denote the canonical projections. Let $x$ be an element of $U$. Then $x=\pi_{1}(x)+\pi_{2}(x)$. By hypothesis, $\pi_{i}(U) \leq U$ for $i=1,2$. Thus $\pi_{i}(x) \in U \cap M_{i}$ for $i=1,2$. Hence $U \leq U \cap M_{1} \oplus U \cap M_{2}$. It follows that $U=U \cap M_{1} \oplus U \cap M_{2}$.

Proposition 2.5. Let $M$ be a nonzero module and let $U$ be a submodule of $M$ such that $f(U) \leq U$ for each $f \in \operatorname{End}_{R}(M)$. If $M$ is $\oplus$-supplemented, then $M / U$ is $\oplus$-supplemented. If, moreover, $U$ is a direct summand of $M$, then $U$ is also $\oplus$-supplemented.

Proof. Suppose that $M$ is $\oplus$-supplemented. Let $L$ be a submodule of $M$ which contains $U$. There exist submodules $N$ and $N^{\prime}$ of $M$ such that $M=N \oplus N^{\prime}$, $M=L+N$, and $L \cap N$ is small in $N$ (Proposition 2.1). By [23, Lemma 1.2(d)], $(N+U) / U$ is a supplement of $L / U$ in $M / U$. Now apply Lemma 2.4 to get that $U=U \cap N \oplus U \cap N^{\prime}$. Thus,

$$
(N+U) \cap\left(N^{\prime}+U\right) \leq\left(N+U+N^{\prime}\right) \cap U+(N+U+U) \cap N^{\prime} .
$$

Hence,

$$
(N+U) \cap\left(N^{\prime}+U\right) \leq U+\left(N+U \cap N+U \cap N^{\prime}\right) \cap N^{\prime} .
$$

It follows that $(N+U) \cap\left(N^{\prime}+U\right) \leq U$ and $((N+U) / U) \oplus\left(\left(N^{\prime}+U\right) / U\right)=$ $M / U$. Then $(N+U) / U$ is a direct summand of $M / U$. Consequently, $M / U$ is $\oplus$-supplemented.

Now suppose that $U$ is a direct summand of $M$. Let $V$ be a submodule of $U$. Since $M$ is $\oplus$-supplemented, there exist submodules $K$ and $K^{\prime}$ of $M$ such that 
$M=K \oplus K^{\prime}, M=V+K$, and $V \cap K \ll K$ (Proposition 2.1). Thus $U=V+U \cap K$. But $U=U \cap K \oplus U \cap K^{\prime}$ (Lemma 2.4), hence $U \cap K$ is a direct summand of $U$. Moreover, $V \cap(U \cap K)=V \cap K$ is small in $K$. Then, $V \cap(U \cap K)$ is small in $U \cap K$ by [23, Lemma 1.1(b)]. Therefore $U \cap K$ is a supplement of $V$ in $U$ and it is a direct summand of $U$. Thus $U$ is $\oplus$-supplemented.

COROLLARY 2.6. Let $M$ be an $R$-module and $P(M)$ the sum of all its radical submodules. If $M$ is $\oplus$-supplemented, then $M / P(M)$ is $\oplus$-supplemented. If, moreover, $P(M)$ is a direct summand of $M$, then $P(M)$ is also $\oplus$-supplemented.

Proof. By Proposition 2.5, it suffices to prove that $f(P(M)) \leq P(M)$ for each $f \in \operatorname{End}_{R}(M)$. Let $N$ be a radical submodule of $M$ and let $f$ be an endomorphism of $M$ and $g$ its restriction to $N$. By [1, Proposition 9.14], $g(\operatorname{Rad}(N)) \leq$ $\operatorname{Rad}(f(N))$. But $\operatorname{Rad}(N)=N$ and $f(N)=g(N)$, hence $f(N) \leq \operatorname{Rad}(f(N))$. Thus, $\operatorname{Rad}(f(N))=f(N)$. This implies that $f(N) \leq P(M)$, and the corollary is proved.

We recall that a module $M$ is called semi-Artinian if every nonzero quotient module of $M$ has nonzero socle. For a module ${ }_{R} M$, we define

$$
\mathrm{Sa}(M)=\sum_{\substack{U \leq M \\ U \text { semi-Artinian }}} U
$$

By [18, Chapter VIII, Section 2, Corollary 2.2], if $R$ is a left Noetherian ring and ${ }_{R} M$ a semi-Artinian left $R$-module, then $M$ is the sum of its submodules of finite length.

If $R$ is a commutative Noetherian ring and $M$ is an $R$-module, then $\operatorname{Sa}(M)=$ $L(M)$, the sum of all Artinian submodules of $M$.

Corollary 2.7. Let $M$ be $a \oplus$-supplemented $R$-module. Then $M / \mathrm{Sa}(M)$ is $\oplus$-supplemented. If, moreover, $\mathrm{Sa}(M)$ is a direct summand of $M$, then $\mathrm{Sa}(M)$ is also $\oplus$-supplemented.

Proof. By Proposition 2.5, it suffices to prove that $f(\mathrm{Sa}(M)) \leq \mathrm{Sa}(M)$ for each $f \in \operatorname{End}_{R}(M)$. Let $U$ be a semi-Artinian submodule of $M$ and let $f$ be an endomorphism of $M$ and $g$ its restriction to $U$. Thus $U / \operatorname{Ker}(g) \cong g(U)$. Hence $f(U) \cong U / \operatorname{Ker}(g)$. But it is easy to check that $U / \operatorname{Ker}(g)$ is a semi-Artinian module. Therefore, $f(U)$ is semi-Artinian.

REMARK 2.8. Let $M$ be a $\oplus$-supplemented module. It is clear that $M / \operatorname{Rad}(M)$ and $M / \operatorname{Soc}(M)$ are also $\oplus$-supplemented (see Proposition 2.5 and [1, Propositions 9.14 and 9.8]).

3. Some properties of finitely generated $\oplus$-supplemented modules. A module $M$ is called supplemented if for any two submodules $A$ and $B$ with $A+B=M, B$ contains a supplement of $A$. 
The proof of the next result is taken from [6, Lemma 2], but is given for the sake of completeness.

LEMMA 3.1. Let $M$ be $a \oplus$-supplemented $R$-module. If $M$ contains a maximal submodule, then $M$ contains a local direct summand.

Proof. Let $L$ be a maximal submodule of $M$. Since $M$ is $\oplus$-supplemented, there exists a direct summand $K$ of $M$ such that $K$ is a supplement of $L$ in $M$. Then for any proper submodule $X$ of $K, X$ is contained in $L$ since $L$ is a maximal submodule and $L+X$ is a proper submodule of $M$ by minimality of $K$. Hence $X \leq L \cap K$ and $X$ is small in $K$ by [12, Lemma 4.5]. Thus $K$ is a hollow module, and the lemma is proved.

Proposition 3.2. If $M$ is a $\oplus$-supplemented module such that $\operatorname{Rad}(M)$ is small in $M$, then $M$ can be written as an irredundant sum of local direct summands of $M$.

Proof. Since $\operatorname{Rad}(M)$ is small in $M, M$ contains a maximal submodule and hence $M$ contains a local direct summand by Lemma 3.1. Let $N$ be the sum of all local direct summands of $M$. If $N$ is a proper submodule of $M$, then there exists a maximal submodule $L$ of $M$ such that $N \leq L$ (see [8, Proposition 9 and Theorem 8]). Let $P$ be a direct summand of $M$ such that $P$ is a supplement of $L$ in $M$. Note that $P$ is a local module (see the proof of Lemma 3.1) and hence it is contained in $N$, so $M=L+P \leq L+N=L$. This is a contradiction. Hence we have $N=M$. Now let $M=\sum_{i \in I} L_{i}$ where each $L_{i}$ is a local direct summand of $M$. Then,

$$
\frac{M}{\operatorname{Rad}(M)}=\sum_{i \in I}\left[\frac{L_{i}+\operatorname{Rad}(M)}{\operatorname{Rad}(M)}\right]
$$

and each

$$
\frac{L_{i}+\operatorname{Rad}(M)}{\operatorname{Rad}(M)} \cong \frac{L_{i}}{L_{i} \cap \operatorname{Rad}(M)}
$$

is simple by [23, Lemma 1.1(c)]. Hence

$$
\frac{M}{\operatorname{Rad}(M)}=\bigoplus_{k \in K}\left[\frac{L_{k}+\operatorname{Rad}(M)}{\operatorname{Rad}(M)}\right]
$$

for some subset $K \subseteq I$. Thus $M=\sum_{k \in K} L_{k}$ since $\operatorname{Rad}(M)$ is small in $M$. Clearly, the sum $\sum_{k \in K} L_{k}$ is irredundant.

COROLLARY 3.3. Let $R$ be a commutative ring and $M$ a finitely generated $R$-module. If $M$ is $\oplus$-supplemented, then $M=H_{1}+H_{2}+\cdots+H_{n}$, where each $H_{i}$ is a local direct summand of $M$ and $n=\operatorname{corank}(M)$. 
Proof. By Proposition 3.2, $M=H_{1}+H_{2}+\cdots+H_{n}$, where each $H_{i}$ is a local direct summand of $M$ and the sum $\sum_{i=1}^{n} H_{i}$ is irredundant. By [16, Corollary 4.6], $M$ is supplemented. Therefore $n=\operatorname{corank}(M)$ by [14, Proposition 1.7] and [19, Lemma 2.36 and Theorem 2.39].

REMARK 3.4. (i) The module $M=\left(R \overline{x_{1}}+R \overline{x_{2}}\right) \oplus R \overline{x_{3}}$ in Example 2.3 is not $\oplus$-supplemented. On the other hand, $M$ can be written as follows: $M=\left(R \overline{x_{1}}+\right.$ $\left.R \overline{x_{2}}\right) \oplus R\left(\overline{x_{1}}-\overline{x_{3}}\right) ; M=\left(R \overline{x_{1}}+R \overline{x_{2}}\right) \oplus R\left(\overline{x_{2}}-\overline{x_{3}}\right)$; and $M=R\left(\overline{x_{1}}-\overline{x_{3}}\right)+R\left(\overline{x_{2}}-\right.$ $\left.\overline{x_{3}}\right)+R \overline{x_{3}}$. Therefore $M$ is an irredundant sum of local direct summands of $M$. However, $M$ is not $\oplus$-supplemented.

(ii) In the same example, we have that $K=R \overline{x_{1}}+R \overline{x_{2}}$ is an indecomposable direct summand of

$$
M=\frac{R x_{1} \oplus R x_{2} \oplus R x_{3}}{R\left(a x_{1}-b x_{2}\right)}=\left(R \overline{x_{1}}+R \overline{x_{2}}\right) \oplus R \overline{x_{3}} .
$$

Then $K$ is not an irredundant sum of local direct summands. This example shows that, in general, a direct summand of a module which is written as an irredundant sum of local direct summands does not have the same property.

Proposition 3.5. Let $M$ be a finitely generated $\oplus$-supplemented module such that $k=\operatorname{corank}(M) \leq 2$. Then $M$ is a direct sum of local modules.

Proof. It is clear that if $k=1$, then $M$ is a local module. Now suppose that $k=2$. Since $M$ is $\oplus$-supplemented, $M$ contains a local direct summand $H$ (Lemma 3.1). Let $K$ be a submodule of $M$ such that $M=H \oplus K$. By [14, Corollary 1.9], we have $\operatorname{corank}(K)=1$ and hence $K$ is a local module (see [19, Proposition 1.11]). Thus $M$ is a direct sum of local modules, as required.

Our next objective is to prove that over a commutative ring, if $M$ is a finitely generated $\oplus$-supplemented module with $\operatorname{corank}(M)=3$, then $M$ is a direct sum of local modules. We first prove the following generalization of [11, Lemma 2.3].

LEMMA 3.6. Let $L_{1}, L_{2}, \ldots, L_{n}$ be indecomposable direct summands of a module $M$ such that $\operatorname{End}_{R}\left(L_{i}\right)$ is a local ring for each $i(1 \leq i \leq n)$. If $L_{i} \not L_{j}$ for all $i \neq j$, then $\sum_{i=1}^{n} L_{i}$ is direct and is a direct summand of $M$.

Proof. We use induction over $n$. Assume that $L_{1}+L_{2}+\cdots+L_{n-1}$ is a direct sum and is a direct summand of $M$ and let $L=L_{1} \oplus L_{2} \oplus \cdots \oplus L_{n-1}$. There exists a submodule $N$ of $M$ such that $M=L \oplus N$. By [20, Proposition 1], $L_{n}$ has the exchange property. Thus, $M=L_{n} \oplus L^{\prime} \oplus N^{\prime}$ for some submodules $L^{\prime}$ and $N^{\prime}$ of $M$ with $L^{\prime} \leq L$ and $N^{\prime} \leq N$. Let $N^{\prime \prime}$ and $L^{\prime \prime}$ be two submodules of $M$ such that $N=N^{\prime} \oplus N^{\prime \prime}$ and $L=L^{\prime} \oplus L^{\prime \prime}$. Hence $M=L^{\prime} \oplus N^{\prime} \oplus L^{\prime \prime} \oplus N^{\prime \prime}$. Therefore, $L_{n} \cong L^{\prime \prime} \oplus N^{\prime \prime}$. This implies that $L^{\prime \prime}=0$ or $N^{\prime \prime}=0$. Hence $L^{\prime}=L$ or $N^{\prime}=N$. Suppose that $N^{\prime}=N$. Thus $L_{n} \oplus L^{\prime} \cong L$. By the Krull-Schmidt-Azumaya theorem, 
every indecomposable direct summand of $L$ is isomorphic to one of the $L_{i}$, $1 \leq i \leq n-1$. It follows that $L_{n}$ is isomorphic to one of the $L_{i}, 1 \leq i \leq n-1$, which is a contradiction. Therefore $L^{\prime}=L$ and $M=L_{n} \oplus L \oplus N^{\prime}$, that is, $M=$ $L_{1} \oplus L_{2} \oplus \cdots \oplus L_{n-1} \oplus L_{n} \oplus N^{\prime}$, and the lemma is proved.

COROLlary 3.7. Suppose that $R$ is commutative or left Noetherian. Let $L_{1}$, $L_{2}, \ldots, L_{n}$ be hollow local direct summands of a module $M$. If $L_{i} \not L_{j}$ for all $i \neq j$, then $\sum_{i=1}^{n} L_{i}$ is direct and is a direct summand of $M$.

Proof. This is a consequence of [4, Theorems 4.1 and 4.2] and Lemma 3.6.

Proposition 3.8. Suppose that $R$ is a commutative ring. Let $M$ be a finitely generated $\oplus$-supplemented module such that all the hollow direct summands of $M$ are isomorphic. Then $M$ is a direct sum of hollow local modules.

Proof. By Proposition 3.2, we can write $M=H_{1}+H_{2}+\cdots+H_{n}$ as an irredundant sum of hollow local direct summands. By hypothesis, $H_{1} \cong H_{2} \cong$ $\cdots \cong H_{n}$. Thus,

$$
\operatorname{Ann}_{R}\left(H_{1}\right)=\operatorname{Ann}_{R}\left(H_{2}\right)=\cdots=\operatorname{Ann}_{R}\left(H_{n}\right)
$$

Hence,

$$
\operatorname{Ann}_{R}(M)=\bigcap_{i=1}^{n} \operatorname{Ann}_{R}\left(H_{i}\right)=\operatorname{Ann}_{R}\left(H_{i}\right) \text { for each } i(1 \leq i \leq n) .
$$

Therefore all hollow local direct summands of $M$ are isomorphic to $R / I$, where $I=\operatorname{Ann}_{R}(M)$. Let $H$ be a local submodule of $M$ such that $H$ is not small in $M$. Since $M$ is $\oplus$-supplemented, there exist submodules $N$ and $N^{\prime}$ of $M$ such that $H+N=M, N^{\prime} \oplus N=M$, and $H \cap N$ is small in $N$ (Proposition 2.1). It follows that $N^{\prime} \cong M / N \cong H /(H \cap N)$. Hence, $N^{\prime}$ is a local module. This implies that $\operatorname{Ann}_{R}\left(N^{\prime}\right)=I$ and $\operatorname{Ann}_{R}(H /(H \cap N))=I$. Thus, the set $\{r \in R \mid r x \in N\}=I$, where $H=R x$. Let $y \in H \cap N$. There exists $\alpha \in R$ with $y=\alpha x$. So $\alpha \in I$ and hence $y=0$ since $I \subseteq \operatorname{Ann}_{R}(H)$. Therefore $H \cap N=0$ and $M=H \oplus N$. It follows that every nonsmall local submodule of $M$ is a direct summand of $M$. Note that corank $(M)<\infty$ (Corollary 3.3). Applying [23, corollary on page 45] and [8, Proposition 9], we get that $M$ is a direct sum of local modules.

COROLLARY 3.9. Let $R$ be a commutative ring and $M$ a finitely generated $\oplus$-supplemented module with corank $(M)=3$. Then $M$ is a direct sum of local modules.

Proof. Let $F_{0}$ be an irredundant set of representatives of the local direct summands of $M$ ( $F_{0}$ is not empty by Lemma 3.1). By Corollary 3.7, $\operatorname{Card}\left(F_{0}\right) \leq 3$. If $\operatorname{Card}\left(F_{0}\right)=3$, then $M$ is a direct sum of local modules (Corollary 3.7). If $\operatorname{Card}\left(F_{0}\right)=2$ and $F_{0}=\left\{L_{1}, L_{2}\right\}$, then there exists a submodule $L_{3}$ of $M$ such that 
$M=L_{1} \oplus L_{2} \oplus L_{3}$ (Corollary 3.7). But corank $(M)=3$. Therefore corank $\left(L_{3}\right)=1$ (see [14, Corollary 1.9]) and hence $L_{3}$ is a local module. If $\operatorname{Card}\left(F_{0}\right)=1$, then $M$ is a direct sum of local modules by Proposition 3.8.

REMARK 3.10. (i) If $M$ is a finitely generated $\oplus$-supplemented module with corank $(M) \leq 2$, then $M$ is completely $\oplus$-supplemented (see [6, Proposition 6] and Proposition 3.5).

(ii) If $R$ is a commutative ring and $M$ a finitely generated $\oplus$-supplemented module with corank $(M)=3$, then $M$ is completely $\oplus$-supplemented (see [6, Corollary 6] and Corollary 3.9).

4. $\oplus$-supplemented modules over commutative principal ideal rings. In this section, the structure of $\oplus$-supplemented modules over a principal ideal ring is completely determined.

Let $R$ be a commutative Noetherian ring. Let $\Omega$ be the set of all maximal ideals of $R$. As in [24, page 53], if $m \in \Omega$ and $M$ is an $R$-module, we denote the $m$-local component of $M$ by $K_{m}(M)=\{x \in M \mid x=0$ or the only maximal ideal over $\operatorname{Ann}_{R}(x)$ is $\left.m\right\}$. We call $M m$-local if $K_{m}(M)=M$ or, equivalently, if $m$ is the only maximal ideal over each $p \in \operatorname{Ass}(M)$. In this case, $m$ is an $R_{m}$-module by the following operation: $(r / s) x:=r x^{\prime}$ with $x=s x^{\prime}(r \in R, s \in R \backslash m)$. The submodules of $M$ over $R$ and over $R_{m}$ are identical.

For $K(M)=\{x \in M \mid R x$ is complemented $\}$, we always have a decomposition $K(M)=\oplus_{m \in \Omega} K_{m}(M)$ and for a complemented module $M$, we have $M=$ $K(M)$ [24, Theorems 2.3 and 2.5].

A principal ideal ring is called special if it has only one prime ideal $p \neq R$ and $p$ is nilpotent [22, page 245].

THEOREM 4.1. Let $R$ be a commutative local principal ideal ring (not necessarily a domain) with maximal ideal $m$.

(i) If $m$ is nilpotent, then every $R$-module is $\oplus$-supplemented.

(ii) If $m$ is not nilpotent, then $R$ is a domain and ${ }_{R} M$ is $a \oplus$-supplemented $R$ module if and only if $M \cong R^{a} \oplus Q^{b} \oplus(Q / R)^{c} \oplus B(1, \ldots, n)$, where $Q$ is the quotient field of $R$ and $B(1, \ldots, n)$ denotes the direct sum of arbitrarily many copies of $R / m, \ldots, R / m^{n}$, for some positive integer $n$.

Proof. (i) Suppose that $m$ is nilpotent. By [1, Theorem 15.20], $R$ is an Artinian principal ideal ring. Thus, every $R$-module is $\oplus$-supplemented by [7, Theorem 1.1].

(ii) Suppose that $m$ is not nilpotent. Then $R$ is not a special principal ideal ring. By [22, Chapter IV, Section 15, Theorem 33], $R$ is a principal ideal domain and the result follows from [12, Proposition A.7].

The proof of the following result can be found in [7, Proposition 2.1].

Proposition 4.2. Let $R$ be a commutative Noetherian ring and $M$ an $R$ module. The following assertions are equivalent: 
(i) $M$ is $\oplus$-supplemented;

(ii) $M=K(M)$ and $K_{m}(M)$ is $\oplus$-supplemented for all $m \in \Omega$.

COROLLARY 4.3. Let $R$ be a commutative principal ideal ring (not necessarily a domain) and $M$ an $R$-module. The following conditions are equivalent:

(i) $M$ is $\oplus$-supplemented;

(ii) (1) the ring $R / p$ is local for all $p \in \operatorname{Ass}(M)$;

(2) if $m \in \Omega$ such that $m R_{m}$ is not nilpotent, then $K_{m}(M) \cong R_{m}^{a} \oplus Q\left(R_{m}\right)^{b}$ $\oplus\left[Q\left(R_{m}\right) / R_{m}\right]^{c} \oplus B_{m}\left(1, \ldots, n_{m}\right)$ (in Mod $\left.-R_{m}\right)$, where $Q\left(R_{m}\right)$ is the quotient field of $R_{m}$ and $B_{m}\left(1, \ldots, n_{m}\right)$ denotes the direct sum of arbitrarily many copies of $R_{m} / m R_{m}, \ldots, R_{m} /\left(m R_{m}\right)^{n_{m}}$, for some positive integer $n_{m}$.

Proof. See Proposition 4.2, [13, Proposition 2.2(b)], and Theorem 4.1.

Proposition 4.4 (see [7, Corollary 2.2]). Let $R$ be a commutative Noetherian ring and $M$ an $R$-module. The following assertions are equivalent:

(i) $M$ is completely $\oplus$-supplemented;

(ii) $M=K(M)$ and $K_{m}(M)$ is completely $\oplus$-supplemented for all $m \in \Omega$.

COROLLARY 4.5. Let $R$ be a commutative principal ideal ring (not necessarily a domain) and $M$ an $R$-module. Then $M$ is $\oplus$-supplemented if and only if $M$ is completely $\oplus$-supplemented.

Proof. By Proposition 4.4 and the proof of Theorem 4.1, it suffices to prove the result for an $R$-module $M$ over a local principal ideal domain $R$ with maximal ideal $m \neq 0$. If $M$ is $\oplus$-supplemented, then $M \cong R^{a} \oplus Q^{b} \oplus(Q / R)^{c} \oplus B(1, \ldots$, $n$ ), where $Q$ is the quotient field of $R$ and $B(1, \ldots, n)$ denotes the direct sum of arbitrarily many copies of $R / m, \ldots, R / m^{n}$ (Theorem 4.1). By [7, Theorem 2.1], $Q^{b} \oplus(Q / R)^{c}$ and $R^{a} \oplus B(1, \ldots, n)$ both are $\oplus$-supplemented. By [6, Corollary 2], $R^{a} \oplus B(1, \ldots, n)$ is completely $\oplus$-supplemented. Now consider the module $Q^{b} \oplus(Q / R)^{c}$. Since $Q$ and $Q / R$ are injective, $\operatorname{End}_{R}(Q)$ and $\operatorname{End}_{R}(Q / R)$ are local rings (see [1, Lemma 25.4]). By [1, Corollary 12.7] and [12, Proposition A.7], $Q^{b} \oplus$ $(Q / R)^{c}$ is completely $\oplus$-supplemented. Hence $Q^{b} \oplus(Q / R)^{c} \oplus R^{a} \oplus B(1, \ldots, n)$ is completely $\oplus$-supplemented (see [7, Corollary 2.1]).

5. Some rings whose modules are $\oplus$-supplemented. A ring $R$ is called a left $V$-ring if every simple left $R$-module is injective. The $\operatorname{ring} R$ is called an $S S I$-ring if every semisimple left $R$-module is injective.

LEMmA 5.1. Let $M$ be a module with $\operatorname{Rad}(M)=0$. Then $M$ is $\oplus$-supplemented if and only if $M$ is semisimple.

Proof. This is clear by [19, Proposition 3.3].

COROLlary 5.2. Let $R$ be a left $V$-ring and $M$ an $R$-module. Then $M$ is $\oplus$ supplemented if and only if $M$ is semisimple. 
Proof. By [3, page 236, Theorem (Villamayor)], for every left $R$-module, $\operatorname{Rad}(M)=0$. Therefore, every $\oplus$-supplemented $R$-module is semisimple (Lemma $5.1)$.

PROPOSITION 5.3. Let $R$ be a ring. The following statements are equivalent:

(i) every $\oplus$-supplemented $R$-module is injective;

(ii) $R$ is a left Noetherian $V$-ring.

Proof. (i) $\Rightarrow$ (ii). Since every semisimple $R$-module is $\oplus$-supplemented, every semisimple $R$-module is injective. Thus $R$ is an SSI-ring. By [3, Proposition 1], $R$ is a left Noetherian $V$-ring.

(ii) $\Rightarrow$ (i). Let $M$ be a $\oplus$-supplemented $R$-module. Since $R$ is a left $V$-ring, $M$ is semisimple (Corollary 5.2). Thus $M$ is an injective $R$-module (see [3, Proposition 1]).

COROLLARY 5.4. Let $R$ be a commutative ring. The following are equivalent:

(i) every $\oplus$-supplemented $R$-module is injective;

(ii) $R$ is semisimple.

Proof. (i) $\Rightarrow$ (ii). It is a consequence of Proposition 5.3 and [3, page 236, Proposition 1 and its first corollary].

(ii) $\Rightarrow$ (i) This application is obvious.

Proposition 5.5. The following assertions are equivalent for a ring $R$ :

(i) for every $R$-module $M, M$ is $\oplus$-supplemented if and only if $M$ is injective;

(ii) $R$ is semisimple.

Proof. (i) $\Rightarrow$ (ii). Suppose that $R$ satisfies the stated condition. By Proposition $5.3, R$ is a left Noetherian $V$-ring. Now, let $M$ be an injective $R$-module. Then $M$ is $\oplus$-supplemented and, since $R$ is a $V$-ring, $M$ is semisimple (Corollary 5.2). Therefore $R$ is a semisimple ring.

(ii) $\Rightarrow$ (i). It is easy to show that every $R$-module is $\oplus$-supplemented and every $R$-module is injective.

REMARK 5.6. If $R$ is a commutative local Noetherian ring having an injective hollow radical $R$-module $H$, then the $R$-module $M=H^{(\mathbb{N})}$ is injective. However $M$ is not $\oplus$-supplemented (see [7, Remark 2.1(3)]). For example, if $R$ is a local Dedekind domain with quotient field $K$, then $K^{(\mathbb{N})}$ is an injective $R$-module which is not $\oplus$-supplemented.

Our next objective is to determine the class of commutative Noetherian rings $R$ with the property that every injective $R$-module is $\oplus$-supplemented. First we prove the following lemma.

LEMMA 5.7. Let $R$ be a quasi-Frobenius ring (not necessarily commutative). Then every injective $R$-module is $\oplus$-supplemented. 
Proof. By [10, Theorem 15.9], every injective $R$-module is projective. Since $R$ is left perfect, every projective $R$-module is $\oplus$-supplemented (see [6, Proposition 13]) and the result is proved.

PROPOSITION 5.8. For a commutative Noetherian ring $R$, the following statements are equivalent:

(i) every injective $R$-module is $\oplus$-supplemented;

(ii) $R$ is Artinian and $E(R / m)$ is a local $R$-module for each maximal ideal $m$ of $R$;

(iii) $R$ is Artinian and $R / I_{m}$ is a quasi-Frobenius ring for each maximal ideal $m$ of $R$, where $I_{m}=\operatorname{Ann}_{R}(E(R / m))$.

Proof. (i) $\Rightarrow$ (ii). By [15, page 53, corollary of Theorem 2.32] and [10, Corollary 3.86], it suffices to prove that $E(R / p)$ is a finitely generated $R$-module for each prime ideal $p$ of $R$. Since $E(R / p)$ is indecomposable (see [15, page 53, corollary of Theorem 2.32]) and $E(R / p)$ is $\oplus$-supplemented, $E(R / p)$ is hollow [6, Proposition 2]. By Remark 5.6, $E(R / p)$ is not radical. Thus, $E(R / p)$ is a local $R$-module.

(ii) $\Rightarrow$ (iii). Let $m$ be a maximal ideal of $R$. Since $E(R / m)$ is a local $R$-module, $E(R / m) \cong R / I_{m}$ where $I_{m}=\operatorname{Ann}_{R}(E(R / m))$. Thus, $R / I_{m}$ is an injective $R$ module. By [9, Theorem 203], $R / I_{m}$ is an injective $\left(R / I_{m}\right)$-module, that is, the ring $R / I_{m}$ is self-injective. Since $R / I_{m}$ is an Artinian ring, $R / I_{m}$ is a quasiFrobenius ring, and the result is proved.

(iii) $\Rightarrow$ (i). Let $M$ be an injective $R$-module. By [15, Theorem 4.5], we can write $M=\oplus_{i \in I} E\left(R / m_{i}\right)$ where the $m_{i}$ are maximal ideals of $R$. Now, $E\left(R / m_{i}\right)$ is an $\left(R / I_{m_{i}}\right)$-module and the $\left(R / I_{m_{i}}\right)$-submodules of $E\left(R / m_{i}\right)$ are the same as the $R$-submodules of $E\left(R / m_{i}\right)$, therefore ${ }_{R}\left(E\left(R / m_{i}\right)\right)$ is $\oplus$-supplemented (see Lemma 5.7 and [9, Theorem 203]). By [6, Proposition 2], $E\left(R / m_{i}\right)(i \in I)$ is a hollow $R$-module. By [1, Corollary 15.21], $\operatorname{Rad}\left(E\left(R / m_{i}\right)\right)$ is small in $E\left(R / m_{i}\right)$. Thus, $E\left(R / m_{i}\right)(i \in I)$ is a local $R$-module. It follows by [1, Corollary 15.21] and [6, Corollary 2] that $M$ is $\oplus$-supplemented.

Proposition 5.9. Let $p$ be a prime ideal of a commutative Noetherian ring $R$ such that $E(R / p)$ is hollow. Then there is a maximal ideal $m$ of $R$ such that

(i) $m$ is the only maximal ideal over $p$;

(ii) $E(R / p)$ has the structure of an $R_{m}$-module;

(iii) the submodules of $E(R / p)$ over $R$ and over $R_{m}$ are identical.

Moreover, as an $R_{m}$-module, $E(R / p)$ is isomorphic to an injective envelope of $R_{m} / S^{-1} p$ where $S=R \backslash m$.

Proof. Suppose that $E(R / p)$ is hollow. Since [13, Proposition 1.1] gives that $E(R / p)$ is $m$-local for some $m \in \Omega, m$ is the only maximal ideal over $p, E(R / p)$ has the structure of an $R_{m}$-module, and the $R_{m}$-submodules of $E(R / p)$ are exactly the $R$-submodules of $E(R / p)$. It remains to show the last assertion. By [15, Proposition 5.5], $E(R / p)$ is injective as an $R_{m}$-module. Now, 
$E(R / p)$ is indecomposable as an $R$-module and its $R_{m}$-submodules are also $R$-submodules so that $E(R / p)$ is also indecomposable as an $R_{m}$-module. Since $\operatorname{Ass}_{R}(E(R / p))=\{p\}$, there is an element $x \in E(R / p)$ such that $\operatorname{Ann}_{R}(x)=p$. But it is easy to check that $\operatorname{Ann}_{R_{m}}(x)=S^{-1} p$ with $S=R \backslash m$ and $S^{-1} p$ is a prime ideal of $R_{m}$. Then $E(R / p)$ is isomorphic to an injective envelope of $R_{m} / S^{-1} p$ by [15, page 53 , Corollary of Theorem 2.32].

Proposition 5.10. Let $p$ be a prime ideal of a commutative Noetherian ring $R$. Then the following are equivalent:

(i) $E(R / p)$ is hollow local;

(ii) $p$ is maximal and $R_{p}$ is a quasi-Frobenius ring.

Proof. (i) $\Rightarrow$ (ii). Suppose that $E(R / p)$ is hollow local. By Proposition 5.9, $E(R / p)$ is $m$-local for some maximal ideal $m$ of $R$ and as an $R_{m}$-module, $E\left(R_{m} / S^{-1} p\right)$ is hollow local, where $S=R \backslash m$. Since $R_{m}$ is Noetherian local, $R_{m}$ is Artinian by [9, Theorem 207]. Hence $S^{-1} p$ is a maximal ideal of $R_{m}$. Thus $S^{-1} p=S^{-1} m$. Therefore $p=m$ is maximal. Moreover, by [15, page 47, Corollary 2], $\operatorname{Ann}_{R_{m}}\left(E\left(R_{m} / S^{-1} m\right)\right)=0$. Then $E\left(R_{m} / S^{-1} m\right) \cong R_{m}$. So $R_{m}$ is self-injective. Therefore $R_{m}$ is a quasi-Frobenius ring.

(ii) $\Rightarrow$ (i). Suppose that $p$ is maximal and $R_{p}$ is a quasi-Frobenius ring. Put $E=E(R / p)$. By [15, Proposition 4.23], $E(R / p)=\sum_{n=1}^{\infty} \operatorname{Ann}_{E}\left(p^{n}\right)$. Then $E$ is $p$ local. Thus $E$ is an $R_{p}$-module and the submodules of $E$ over $R$ and over $R_{p}$ are identical. The proof of Proposition 5.9 shows that, as an $R_{p}$-module, $E$ is isomorphic to $E\left(R_{p} / p R_{p}\right)$, where $p R_{p}$ denotes the unique maximal ideal of $R_{p}$. On the other hand, since $R_{p}$ is a self-injective Artinian local ring, $E\left(R_{p} / p R_{p}\right)$, as an $R_{p}$-module, is isomorphic to $R_{p}$ (see [10, Theorem 15.27]). Hence $E\left(R_{p} / p R_{p}\right)$ is a local $R_{p}$-module. Consequently, $E$ is a local $R$-module.

LEMMA 5.11. Let $R$ be a commutative ring. If $R$ is Noetherian and $R_{m}$ is quasi-Frobenius for every maximal ideal $m$ of $R$, then $R$ is quasi-Frobenius.

Proof. Let $m$ be a maximal ideal of $R$. Since $R_{m}$ is quasi-Frobenius, then $R_{m}$ is Artinian and so $m R_{m}$, the maximal ideal of $R_{m}$, is a minimal prime ideal. Therefore $m$ is a minimal prime ideal of $R$. The $\operatorname{ring} R$ is Noetherian and every prime ideal is maximal, hence $R$ is Artinian. Let $R=R_{1} \times \cdots \times R_{t}$ where each $R_{i}$ is Artinian and local. Since each $R_{i}$ is a localization of $R$, then $R_{i}$ is quasiFrobenius for each $i=1, \ldots, t$. It is not difficult to see that a finite product of rings is quasi-Frobenius if and only if each factor is quasi-Frobenius (see [10, Theorem 15.27]). Hence $R=R_{1} \times \cdots \times R_{t}$ is quasi-Frobenius.

THEOREM 5.12. For a commutative Noetherian ring $R$, the following statements are equivalent:

(i) every injective $R$-module is $\oplus$-supplemented;

(ii) $R_{m}$ is quasi-Frobenius for each maximal ideal $m$ of $R$;

(iii) $R$ is quasi-Frobenius. 
Proof. (i) $\Rightarrow$ (ii). It is a consequence of Propositions 5.8 and 5.10.

(ii) $\Rightarrow$ (iii). It is clear by Lemma 5.11 .

(iii) $\Rightarrow$ (i). See Lemma 5.7 .

Proposition 5.13. For a $V$-ring, the following statements are equivalent:

(i) $R$ is semisimple;

(ii) every $R$-module is $\oplus$-supplemented.

Proof. (i) $\Rightarrow$ (ii). It is obvious.

(ii) $\Rightarrow$ (i). Suppose that every $R$-module is $\oplus$-supplemented. By Corollary 5.2, every $R$-module is semisimple. Thus $R$ is semisimple, as required.

\section{REFERENCES}

[1] F. W. Anderson and K. R. Fuller, Rings and Categories of Modules, Graduate Texts in Mathematics, vol. 13, Springer-Verlag, New York, 1974.

[2] N. Bourbaki, Éléments de Mathématique. Fasc. XXXI. Algèbre Commutative. Chapitre 1: Modules Plats, Actualités Scientifiques et Industrielles, no. 1314, Hermann, Paris, 1965 (French).

[3] K. A. Byrd, Rings whose quasi-injective modules are injective, Proc. Amer. Math. Soc. 33 (1972), 235-240.

[4] P. Fleury, Hollow modules and local endomorphism rings, Pacific J. Math. 53 (1974), 379-385.

[5] A. Harmancı, D. Keskin, and P. F. Smith, On $\oplus$-supplemented modules, Acta Math. Hungar. 83 (1999), no. 1-2, 161-169.

[6] A. Idelhadj and R. Tribak, A dual notion of CS-modules generalization, Algebra and Number Theory (Fez) (M. Boulagouaz and J.-P. Tignol, eds.), Lecture Notes in Pure and Appl. Math., vol. 208, Marcel Dekker, New York, 2000, pp. 149-155.

[7] , Modules for which every submodule has a supplement that is a direct summand, Arab. J. Sci. Eng. Sect. C Theme Issues 25 (2000), no. 2, 179189.

[8] T. Inoue, Sum of hollow modules, Osaka J. Math. 20 (1983), no. 2, 331-336.

[9] I. Kaplansky, Commutative Rings, revised ed., The University of Chicago Press, Illinois, 1974.

[10] T. Y. Lam, Lectures on Modules and Rings, Graduate Texts in Mathematics, vol. 189, Springer-Verlag, New York, 1999.

[11] S. H. Mohamed and B. J. Müller, Direct sums of dual continuous modules, Math. Z. 178 (1981), no. 2, 225-232.

[12] _ Continuous and Discrete Modules, London Mathematical Society Lecture Note Series, vol. 147, Cambridge University Press, Cambridge, 1990.

[13] P. Rudlof, On the structure of couniform and complemented modules, J. Pure Appl. Algebra 74 (1991), no. 3, 281-305.

[14] B. Sarath and K. Varadarajan, Dual Goldie dimension. II, Comm. Algebra 7 (1979), no. $17,1885-1899$.

[15] D. W. Sharpe and P. Vámos, Injective Modules, Cambridge Tracts in Mathematics and Mathematical Physics, no. 62, Cambridge University Press, London, 1972.

[16] P. F. Smith, Finitely generated supplemented modules are amply supplemented, Arab. J. Sci. Eng. Sect. C Theme Issues 25 (2000), no. 2, 69-79. 
[17] P. F. Smith and A. Tercan, Generalizations of CS-modules, Comm. Algebra 21 (1993), no. 6, 1809-1847.

[18] B. Stenström, Rings of Quotients. An Introduction to Methods of Ring Theory, Die Grundlehren der Mathematischen Wissenschaften, vol. 217, SpringerVerlag, New York, 1975.

[19] K. Varadarajan, Dual Goldie dimension, Comm. Algebra 7 (1979), no. 6, 565-610.

[20] R. B. Warfield Jr., A Krull-Schmidt theorem for infinite sums of modules, Proc. Amer. Math. Soc. 22 (1969), 460-465.

[21]__ Decomposability of finitely presented modules, Proc. Amer. Math. Soc. 25 (1970), 167-172.

[22] O. Zariski and P. Samuel, Commutative Algebra. Vol. 1, Graduate Texts in Mathematics, no. 28, Springer-Verlag, New York, 1975.

[23] H. Zöschinger, Komplementierte Moduln über Dedekindringen, J. Algebra 29 (1974), 42-56 (German).

[24] _ Gelfand ringe und koabgeschlossene Untermoduln [Gelfand rings and coclosed submodules], Bayer. Akad. Wiss. Math.-Natur. Kl. Sitzungsber. 3 (1982), 43-70 (German).

[25] _ Komplemente als direkte Summanden. II [Complements as direct summands. II], Arch. Math. (Basel) 38 (1982), no. 4, 324-334 (German).

A. Idelhadj: Département de Mathématiques, Faculté des Sciences de Tétouan, Université Abdelmalek Essaâdi, B.P 21.21 Tétouan, Morocco

E-mail address: ide1hadj_a@ahotmai 1.com

R. Tribak: Département de Mathématiques, Faculté des Sciences de Tétouan, Université Abdelmalek Essaâdi, B.P 21.21 Tétouan, Morocco

E-mail address: tribak12@yahoo.com 


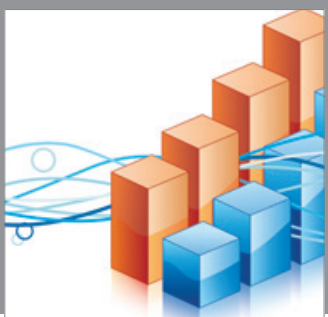

Advances in

Operations Research

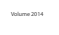

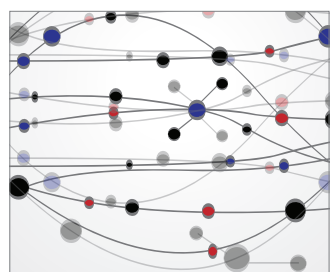

\section{The Scientific} World Journal
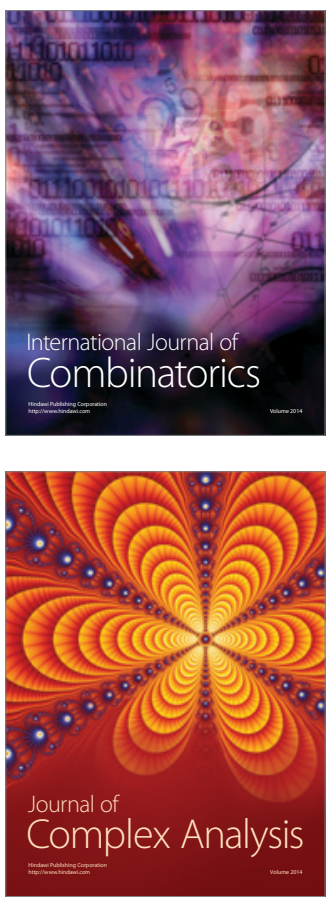

International Journal of

Mathematics and

Mathematical

Sciences
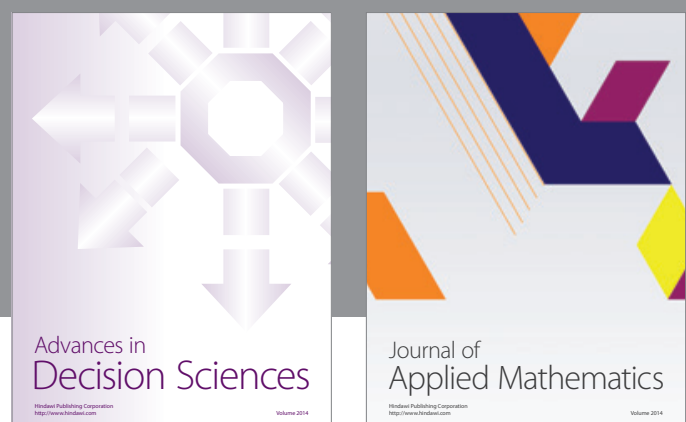

Journal of

Applied Mathematics
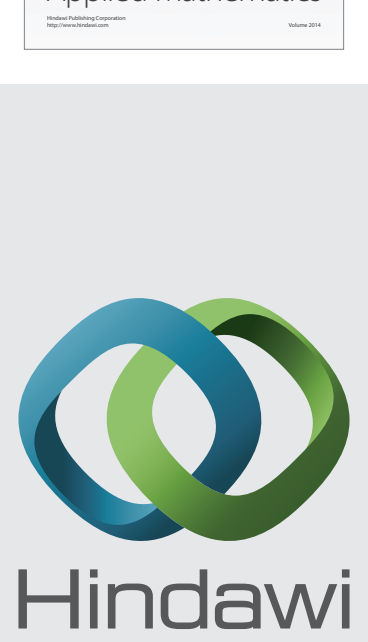

Submit your manuscripts at http://www.hindawi.com
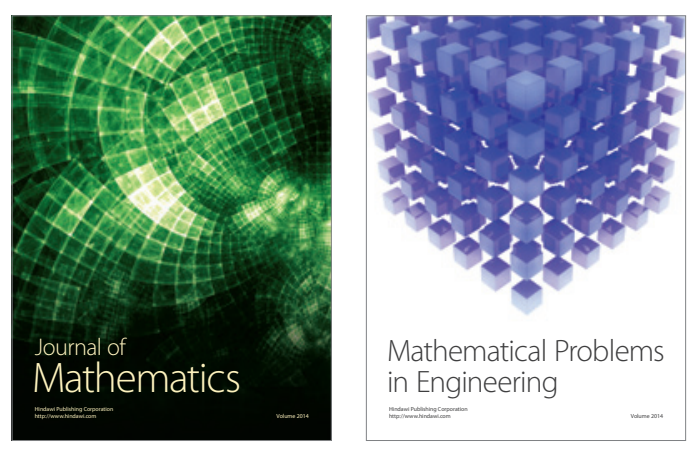

Mathematical Problems in Engineering
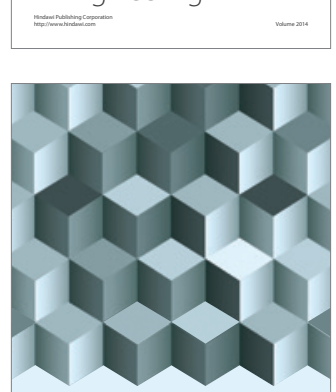

Journal of

Function Spaces
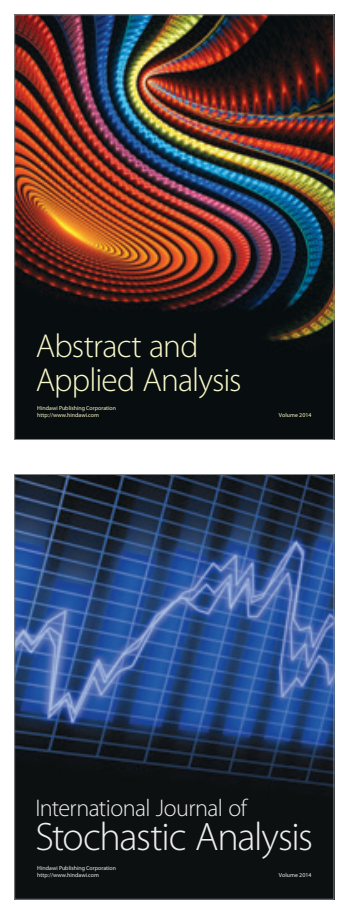

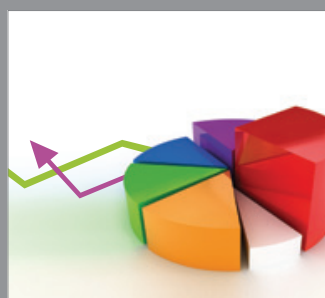

ournal of

Probability and Statistics

Promensencen
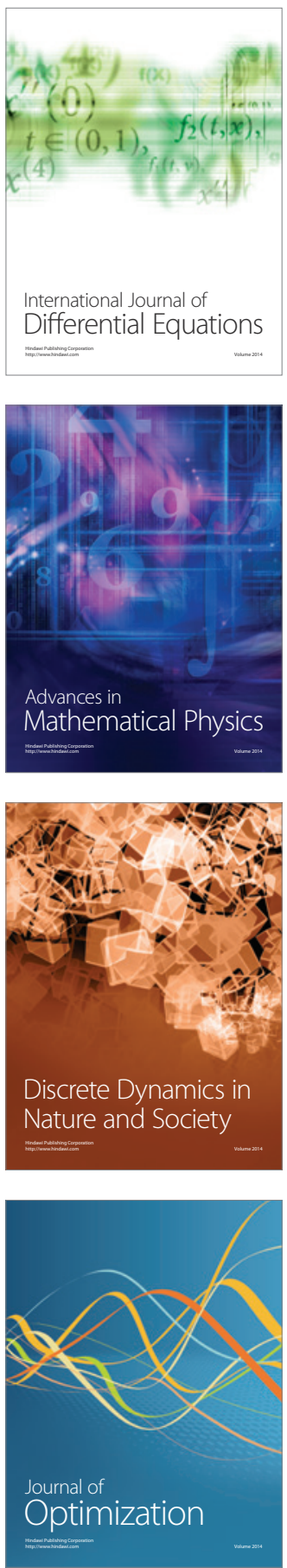